\title{
Proceeding
}

Asia Pacific Conference on Performance Analysis of Sport, 21-24 April 2014. Langkawi, Malaysia

\section{Defense performance analysis: social network analysis}

\author{
KOH SASAKI
}

Nagoya University, Japan

\begin{abstract}
Sasaki, K. (2015). Defense performance analysis: social network analysis. J. Hum. Sport Exerc., 9(Proc2), pp.S747-S752. This article discusses about the defense performance analysis of rugby union and introduces some analysis approaches. Rugby World Cup is a rugby union tournament contested every four years between the top international teams and is one of the largest international sporting events in the world, like FIFA-WC, The Olympic Games and the Tour de France. The Seventh tournament is going to held in Japan in 2019. After watching the three evidences, we will present some analytical approaches. Data were derived from 20 matches of close and balanced scores (under 20 points: results from cluster analysis according to game final score differences of 40 pool matches in 2011RWC). In these day's rugby union games, the point balance under 20 points might be comparatively valid value as closed game. Double tackle contributor's mapping by force-directed placement (degree centrality) at 2011 Rugby World Cup close games in pool matches. Centering Resonance Analysis would be one strong tool for understanding the total relationship structure of network graph, especially when the numbers of vertex has increased and complicated. Network analysis would be one cruel for understanding the complex sport scenes. We hope to keep discussing over the 2019 RWC / 2020 Tokyo Olympic games. we desire for making sport family in whole world and of course in Asian Friends. Key words: RUGBY, PERFORMANCE ANLYSIS, DEFENSE.
\end{abstract}

Corresponding author. Nagoya University, 1 Furocho, Chikusa Ward, Nagoya, Aichi Prefecture 464-0814, Japan.

E-mail: sasaki@htc.nagoya-u.ac.jp

Asia Pacific Conference on Performance Analysis of Sport, 21-24 April 2014. Langkawi, Malaysia.

JOURNAL OF HUMAN SPORT \& EXERCISE ISSN 1988-5202

(c) Faculty of Education. University of Alicante

doi:10.14198/jhse.2015.10.Proc2.16 


\section{INTRODUCTION}

This article discusses about the defense performance analysis of rugby union and introduces some analysis approaches. Rugby World Cup is a rugby union tournament contested every four years between the top international teams and is one of the largest international sporting events in the world, like FIFA-WC, The Olympic Games and the Tour de France. The Seventh tournament is going to held in Japan in 2019. After watching the three evidences, we will present some analytical approaches.

The first evidence:

\section{Defense performance Analysis zocial Network Analysis}

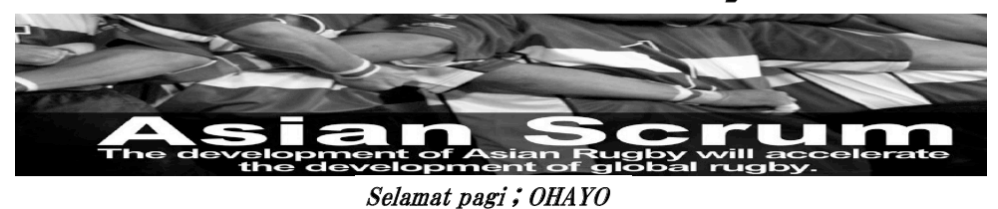

$6.9(2003) \Rightarrow 6.2(2007) \Rightarrow 5.5(2011)$

the numbers of tries per match in the Rugby World Cup

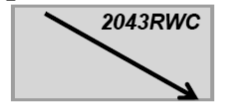

///suggested the defensive capabilities have improved?

Figure 1. Understanding the game results.

What are these values of "6.9, 6.2 and 5.5?" Those were decreasing. These are the number of the tries in RWC(2003, 2007 and 2011). On this tendency, you can anticipate in 2015, 4.8, and $20194.1 \ldots$ Finally, you can anticipate the number of the tries in RWC will be ZERO, in 2043. It suggests the defense capabilities have been improved, or not.

Second evidence:

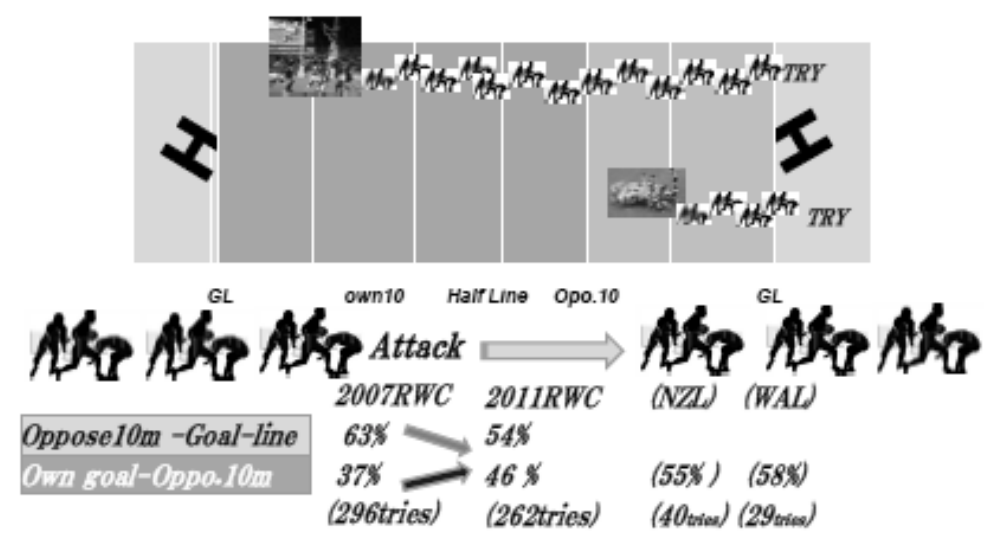

Start of a possession that leaIs to a try in Ruglyy Wort! Gull 2011 comuaring 2007.

Figure 2. This characteristic might suggest that the tries progressed with high level fitness during the offensive game and also with high level defensive performance as a crucial factor. 
These are the start areas of the possession that leads to a try in RWC 2011 comparing 2007. The tries ratio of the area near the goal line was $63 \%$ in 2007, and the tries ratio of the comparative far area where need more effort to the goal because the distance was $37 \%$. It might be logical values. However, these values change in 2011. The ratio of the goal near side decreased on 10 points. So, the ratio of the far area increased around 10 points. World champion team NZ all blacks got 55\% of tries from far area. The highest team of the tries from the far area was wales, the fourth grade in $2011 \mathrm{RWC}$. Wales got $58 \%$ of the tries from the far side. This characteristic might suggest that the tries progressed with high level fitness during the offensive game and also with high level defensive performance as a crucial factor.

\section{Third evidence:}

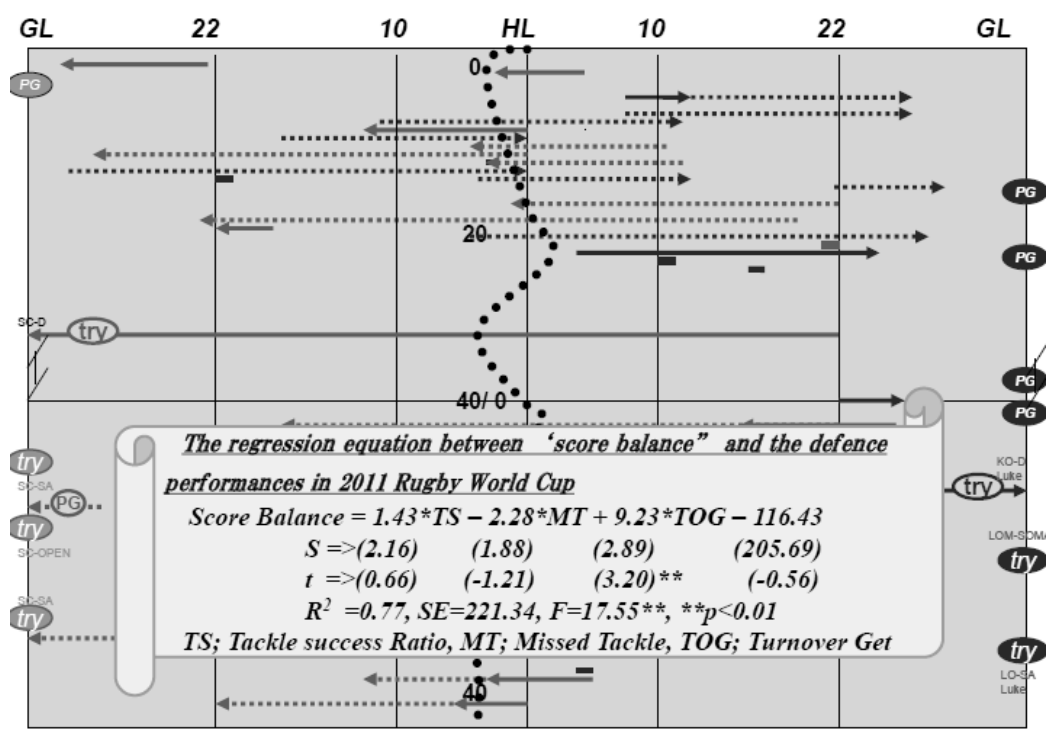

Figure 3. Turn-over gain (run $\rightarrow$ kick $\rightarrow$ ) ad score balance $(\bullet \bullet \bullet \bullet)$ in ime series at one game 2007RWC

The pitch shows the turnover gain series in figure 3 . The vertical line shows time series from kick off to 80 minute game end. The allows show the turnover points and gain after turnover. The solid lines show running gain after turnover. The dot lines show the kick gain after turnover. The black big dot wave indicates the point balance on the scale as Halfline-10m means 10 point balance. This figure shows the turnover appearance on the 80 minute game and the point balance. The final result was 4 point lead by the side which attacks from right to left direction. The oppose team which attacks from left to right direction, after 70 minutes, the turnover appearance decreased compare to the silver side.

By using this defense performance analysis on 2007 RWC, we analyzed 2011 RWC. For understanding the defence performance towards the final score balance, we executed the regression analysis. Independent variables; 'tackle success ratio: like 80-90 (\%)' it shows TS, 'frequencies of the missed tackles' it shows MT, and 'frequencies of crucial turnover get 'TOG, Dependent variable is the 'final score balances". High regression values, 77, were obtained $(\mathrm{R} 2=0.77, \mathrm{SE}=221.34, \mathrm{~F}=17.75, p<0.01)$. The findings presented in this eqation indicate that the three independent variables of defence performance were contributing factors for final score balance. One point up in the 'tackle success ratio' (for example: $79 \%$ to $80 \%$ ) could contribute positive 1.43 to the final score balance. One crucial 'missed tackle' could influence negative 2.28 to final score balance. And one crucial 'turnover get' contribute positive 9.23 points 
to the final score balance. Furthermore, a statistically significant correlation was found between the independent variable 'frequencies of turnover get' TOG and the index 'final score balance'. Practically speaking, the index of 'turnover get' comparing with tackled situations (tackle success or not, missed tackle or not) would have a more positive effect towards the final score balance. So the next focus was on tackle turnover situations.

Its break time.

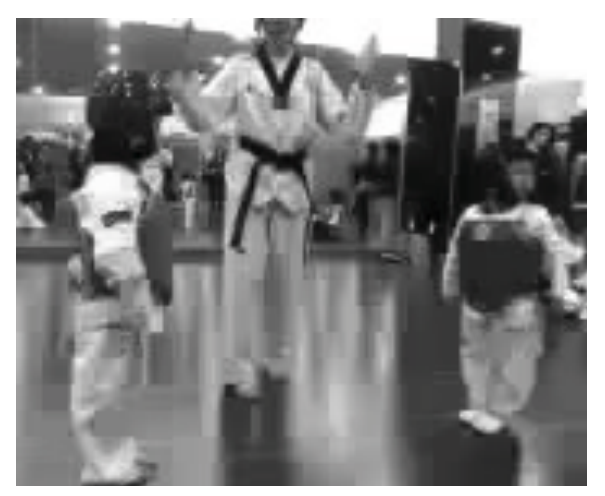

Figure 4. Break: Future SAMURAI challenges the front and back kick

Kicking performance and footwork are very important to all sports. We JOC \& JRFU have developing some martial art program. You can watch the blue boy he is the future samurai's kicking effort and his potential. One essence of the Performance analysis is to enjoy to watching the future generation Kids are always our treasure. We have a mission to make a road for future samurai.

\section{MATERIAL AND METHODS}

After the IRB 2009 rules amendment for advantage to the attacking side, the discussion about a defense should include the more physical intensiveness of co-operation between team-mates. Greenwood pointed out the defense team-mates must move up as one man and make pressure by balanced aggression (2003, p281). However, there has been little descriptive study about two man tackle turnover.

Data were derived from 20 matches of close and balanced scores (under 20 points: results from cluster analysis according to game final score differences of 40 pool matches in 2011RWC). In these day's rugby union games, the point balance under 20 points might be comparatively valid value as closed game.

Network analysis would clarify the evidence of dynamic balance mechanism of defensive co-operation of team mates. Network analysis has been developed in communication-network studies as a graph theory.

The network has structure of both the vertex (players' positions) and the edge line (co-operation between team-mates in match; two man tackle turnover). It shows which position plays a central role of two man tackle turnover. To understand the two man positional relation structure, the frequencies of two man tackle turnover were plotted in the adjacent matrix $(15 * 15)$.

There are many indicators of study about the centrality of network. This article picks up two of them. These are a "density" and "eigenvector." Density centrality means the frequency of double tackled turnover. 
Eigenvector Centrality includes the centrality of the connected neighbors. The purpose was to position the vertexes of a graph in two-dimensional space so that all the edges were of more or less equal length (and there were as few crossing edges as possible).

\section{RESULTS AND DISCUSSION}

Double tackle contributor's mapping by force-directed placement (degree centrality) at 2011 Rugby World Cup close games in pool matches (T; total, W; winners, L; losers, bold black lines; more than 5 times double tackle turnover partners)

Main double tackle contributors which mapped centrally in the team positions graph were "8","6","12","7".

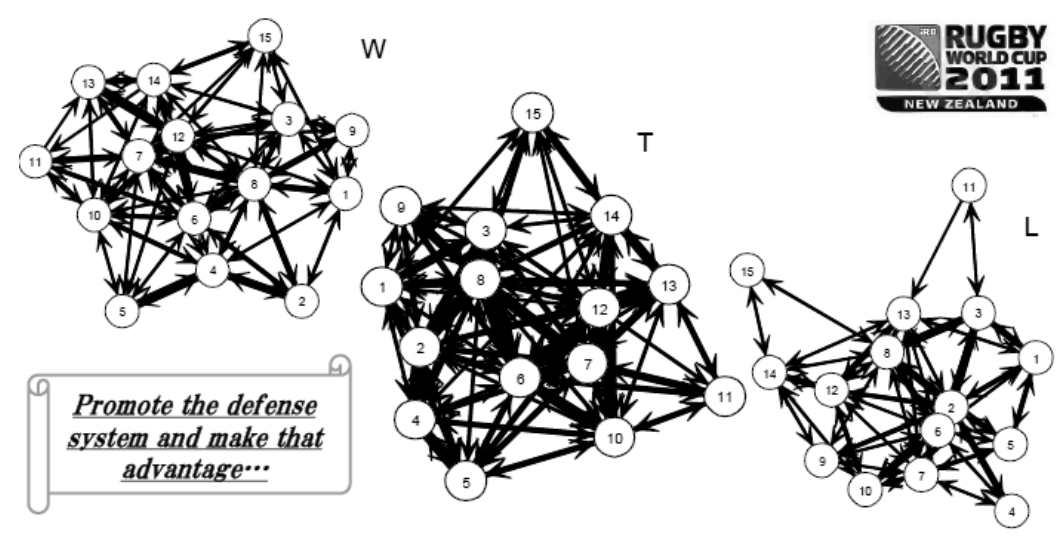

Figure 5. Main double tackle contributors which mapped centrally in the team positions graph were "8", "6", "12", "7". Double tackle contributor's mapping by force-directed placement (degree centrality) at 2011 Rugby World Cup close games in pool matches (T: total ; W: winners; L: losers; bols black lines; more than 5 times double tackle turnover partners). These figure are applied from Sasaki (2013, Performance Analysis IX)

We should promote the defense system and make that advantage.

Network Graph analysis can verify the difference or the similarity between some graphs. Centering Resonance Analysis would be one strong tool for understanding the total relationship structure of network graph, especially when the numbers of vertex has increased and complicated. Now we are trying to make 3 dimensional graph of network analysis. 


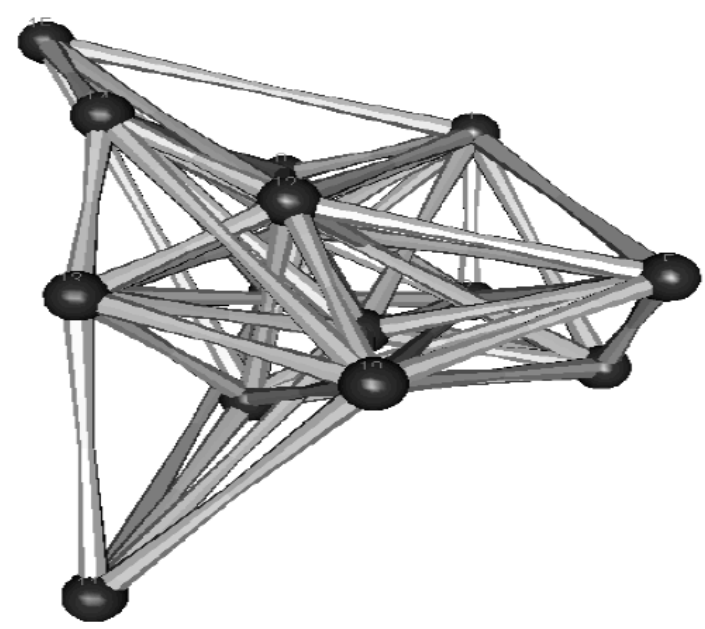

Figure 6. Three dimension plot of the double tackle contributors which mapped centrally in the team positions

It might show the networking dynamics.

Network analysis would be one cruel for understanding the complex sport scenes. We hope to keep discussing over the 2019 RWC / 2020 Tokyo Olympic games. we desire for making sport family in whole world and of course in Asian Friends.

(Terima kasih ; Arigato, Jumpa lagi: mataaimasho)

${ }^{*}$ This article was modified from Sasaki (2013a)

\section{REFERENCES}

1. Duch, J., Waitzman, J.S., \& Nunes Amaral, L.A. (2010). Quantifying the performance of individual players in a team activity. PlosOne, 5(6), e10937.

2. Sasaki, K., Takumi, Y., Jun, M., \& Yuichi U. (2013). Defence performance analysis of rugby union in Rugby World Cup 2011: network analysis of the turnover contributors. In D.M. Peters \& P. O' Donoghue (Eds.). Performance Analysis of Sport IX (pp.94-99). Oxford: Routledge.

3. Sasaki, K., Kayoko, K., Takumi, Y., Yuichi, U., Takashi, K., \& Ichiro K. (2013b). Cognitive societalhuman values of sports: after the 2011 disaster of Japan. Social Sciencies, 2(1), pp.1-6. 